\title{
Development of the Biopolymeric Optical Planar Waveguide with Nanopattern
}

\author{
Seung H. Yoon ${ }^{1}$, Won T. Jeong ${ }^{1}$, Kyung C. Kim ${ }^{1}$, Kyung J. Kim², Min C. Oh ${ }^{2}$, Sang M. Lee \\ ${ }^{1}$ Mechanical Engineering, Pusan National University, Pusan, Korea; ${ }^{2}$ Electrical Engineering, Pusan National University, Pusan, Ko- \\ rea; ${ }^{3}$ Pusan National University, Pusan, Korea. \\ Email: smlee@pnu.edu
}

Received May $18^{\text {th }}$, 2011; revised June $15^{\text {th }}, 2011$; accepted June $25^{\text {th }}, 2011$.

\begin{abstract}
This paper demonstrates for fabricating the biopolymric optical planar waveguide. Gelatin and chitosan were mixed with ratio of 9 to 1 and stirred at $70{ }^{\circ} \mathrm{C}$ with $1300 \mathrm{rpm}$. The blended biopolymer was spincoated on silicon substrate with $500 \mathrm{rpm}$ and then dried in the oven at $50{ }^{\circ} \mathrm{C}$. The refractive indices of the prepared biopolymer clad and core layers of the waveguide were measured by the ellipsometry. The measured refractive indices of the two layers were obtained to be 1.516 and 1.52, respectively. The nanograting was successfully imprinted on surface of the biopolymeric waveguide.
\end{abstract}

Keywords: Biopolymeric Optical Waveguide, Nanograting

\section{Introduction}

This work aims to develop highly sensitive all-biopolymeric planar Bragg grating biosensor. Biopolymer [1-4] is biocompatible material which is developed by blending the chitosan and gelatin [5]. The optical planar waveguide is composed of a clad and core layers. The refractive index of the core layer is slightly higher than that of the clad layer so that light can be guided in the waveguide. The Bragg grating is printed on the developed optical planar waveguide by using the nanoimprint technique.

\section{Theory}

This section provides the basic mathematical foundation that will be used to design and perform data analysis of the proposed planar waveguide grating sensor. This section starts by providing the expressions needed to calculate the propagation constants for the slab waveguide in terms of the waveguide geometric and optical properties.

Then the coupled mode equations used to determine the grating properties are presented. The waveguide geometry of interest in this paper is illustrated in Figure $\mathbf{1}$, and consists of a biopolymer core bounded by air above and a biopolymer clad below. This waveguide is fabricated on a silicon substrate. The refractive indices of air, clad and core layers are denoted by $n_{1}, n_{2}$, and $n_{3}$

*Korean Government (NRF-2009).

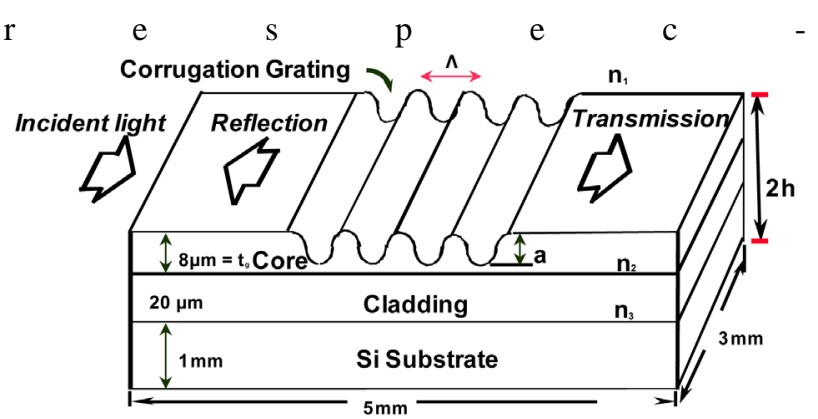

Figure 1. Schematic of the slab waveguide with surface corrugation grating.

tively and the core thickness is given by $t_{g}$. Also seen in Figure 1 are the corrugations of pitch and depth a. The most important parameter in the design of Bragg grating in slab waveguide is the effective index. This effective index is found in the usual way [6] by solving the wave equation and applying the continuity boundary conditions at the respective core/cladding interfaces of the waveguide shown in Figure 1. The guided modes have a propagation constant $\beta_{s}$ such that $k_{0} n_{3}<\beta_{s}<k_{0} n_{2}$, where $n_{1}<n_{3}$. This solution process leads to the following transcendental equation that yields the propagation constant:

$$
\tanh t_{g}=\frac{p+q}{h\left(1-\frac{p q}{h^{2}}\right)}
$$


where $h=\left(n_{y}^{2} k_{0}^{2}-\beta_{s}^{2}\right)^{1 / 2}, q=\left(\beta_{s}^{2}-n_{1}^{2} k_{0}^{2}\right)^{1 / 2}$, $p=\left(\beta_{s}^{2}-n_{1}^{2} k_{0}^{2}\right)^{1 / 2}$ and $k_{0} \approx \omega / c=2 \pi / \lambda$. Given a set of refractive indices $n_{1}, n_{2}$, and $n_{3}$ and the waveguide thickness, $t_{g}$, of the planar waveguide, and the source wavelength, $\lambda$, Equation (1) in general yields a number of solutions for the propagation constant, $\beta_{s}$. However, the source wavelength and the waveguide thickness are restricted in the present study such that only one propagation mode is supported, and therefore Equation (1) has only one solution of interest. As a result, the effective index of the planar waveguide is given by $n_{\text {eff }}=\beta_{s} \lambda / 2 \pi$. The corrugated structure into the waveguide leads to a corresponding periodic perturbation of the refractive index distribution. Each groove of the grating acts like a weak mirror, and the cumulative effect of all of the weak reflectors results in a very strong combined reflection centered on what is known as the Bragg wavelength. The Bragg wavelength is related to the effective index calculated above and the grating period, $\Lambda$, by [6]

$$
\lambda_{b}=2 n_{\text {eff }}
$$

which when expressed in terms of the propagation constant is given by

$$
\lambda_{b}=\frac{\beta_{s} \lambda \Lambda}{2}
$$

where $\lambda_{b}$ is the Bragg wavelength and $\lambda$ is the central wavelength of the optical source.

\section{Experiment}

\subsection{The Production of Biopolymer through Blending of Chitosan and Gelatin}

The biopolymer was developed in this study by blending chitosan and gelatin. The buffer solution which was composed of sodium acetate and acetic acid dissolved chitosan and gelatin in liquid state. In order to control the refractive index of the material, the ratios of chitosan to gelatin were 1:9, 2:8, 5:5, 8:2 and 9:1. Figure 2 shows procedures for blending the biopolymer.

There are issues addressed in the process for dissolving the biopolymers. As the first issue, viscosity of chitosan in liquid state increases when the amount of chitosan in the buffer increases. When blending chitosan with gelatin in the liquid state, conglomeration of chitosan may occur and accordingly it causes surface quality. Another issue is that chitosan in acetic acid is not completely insoluble and remained to be particle state.

To measure the refractive index of the sample, surface of the sample must be kept clean and flat. In order to mitigate the problems mentioned above, dissolving the amount of chitosan was controlled to prevent increase in viscosity of chitosan solution, and chitosan particles exi-

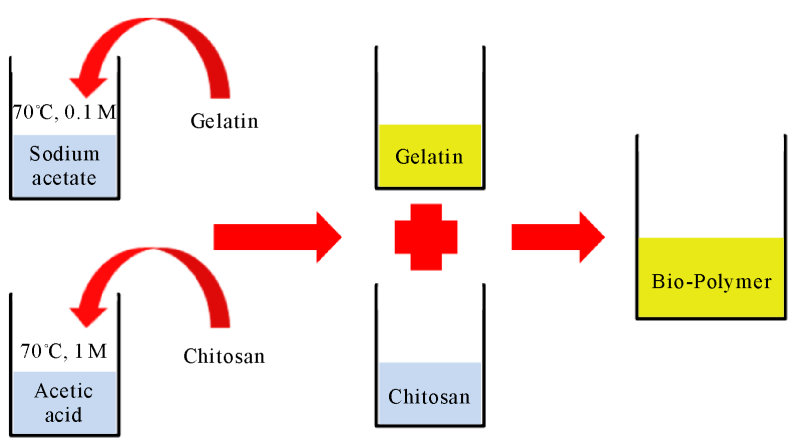

Figure 2. Schematic of blending process of a biopolymer.

sting in the solution have been removed using the centrifuge.

\subsection{Fabrication of Biopolymeric Optical Waveguide}

The ratio of chitosan and gelatin was varied to obtain the slightly different refractive indices of the biopolymers which consisted of the biopolymeric waveguide. The biopolymer to be used in core layer was mixed with the ratio of chitosan of $0.05 \mathrm{~g}$ and gelatin of $1.03 \mathrm{~g}$. The clad layer was mixed with the ratio of chitosan of $0.03 \mathrm{~g}$ and gelatin of $1.05 \mathrm{~g}$. The biopolymer was spincoted on the silicon wafer with $550 \mathrm{rpm}$ for the $30 \mathrm{~s}$ and then baked for 5 hours at $50^{\circ} \mathrm{C}$. The ellipsometer was used to measure the refractive index of the spincoted film of the biopolymer.

\subsection{Optical Butt Coupling}

The optical planar waveguide was butt-coupled to investigate the light propagation into the waveguide. The light is incident into the optical fiber which was butt coupled to the waveguide, and then the light transmitted through the waveguide was monitored by the CCD camera, as shown in Figure 3.

The Bragg grating which will be imprinted on the biopolymeric planar waveguide obtained in this study is designed by using the Equation (2). Figure 4 shows schematic of the optical waveguide, indicating that the grating pitch was determined by the effective refractive index of and thickness of waveguide.

\subsection{Imprint of Bragg Grating on the Biopolymric Waveguide}

Holographic grating, as shown in Figure 5, is imprinted on the photoresist spincoated on the silicon substrate with grating period obtained by calculating the optical waveguide. PDMS grating was molded on the PDMS by using the photoresist grating on the silicon substrate. PDMS grating mold production process and a grating imprinting technique are shown in Figures 6 and 7. 


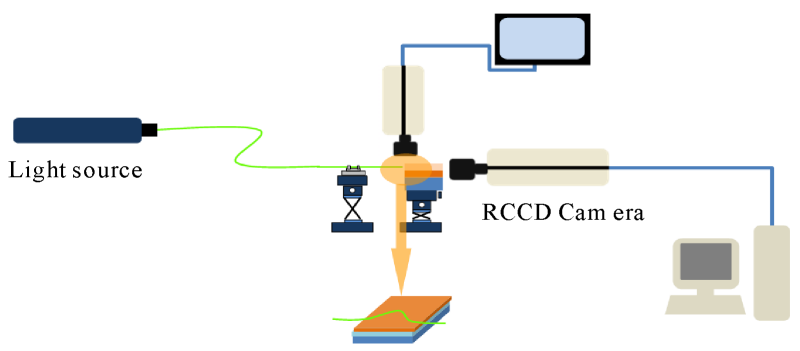

Figure 3. Schematic of optical butt coupling setup.

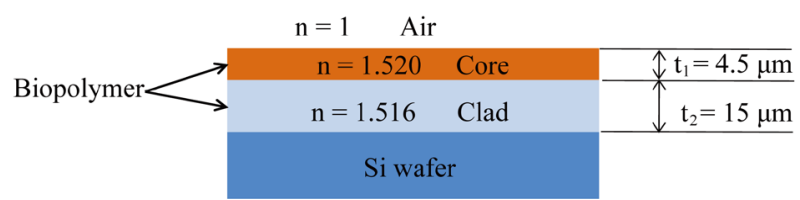

Figure 4. Schematic of the optical waveguide.

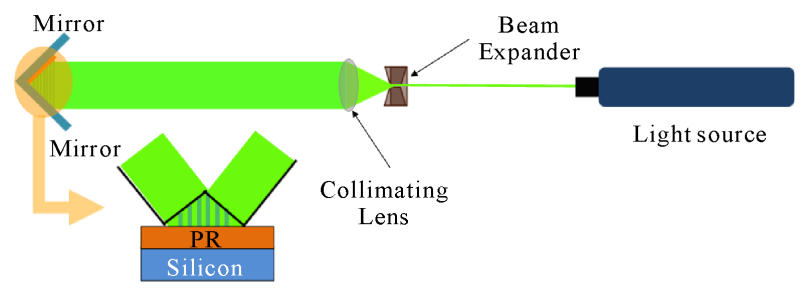

Figure 5. Schematic of holographic lithography of the grating.

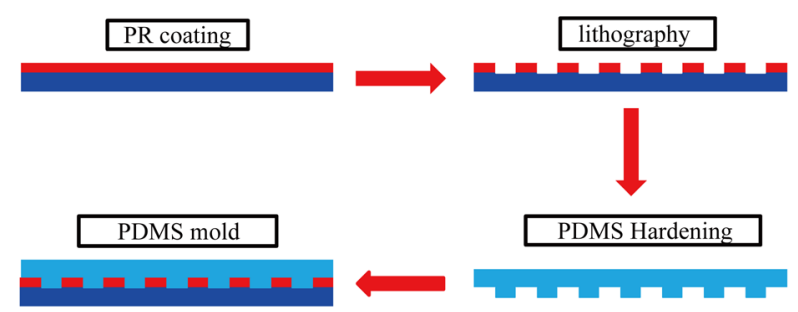

Figure 6. Schematic of PDMS grating mold.

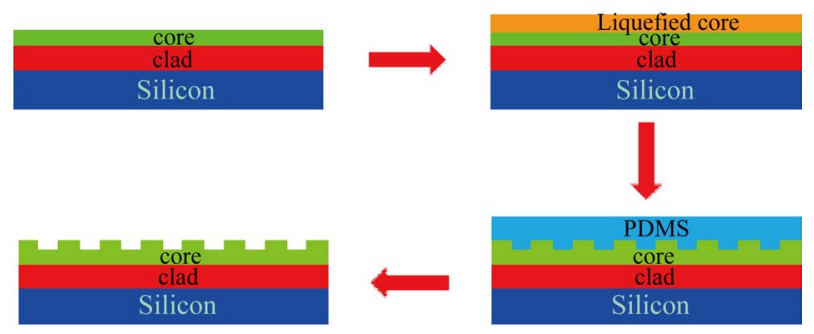

Figure 7. Schematic of grating imprinting process.

\section{Results and Discussion}

The ratio of blending of chitosan and gelatin makes the refractive index of the biopolymeric material changed. The blended biopolymer was coated on the substrate with the proper velocity of spincoating and time. The condi- tion for spincoating is shown in Table 1. The thickness of the spincoated layer of the biopolymer was measured to be $300 \mathrm{~nm}-800 \mathrm{~nm}$.

However, the biopolymer layer was spincoated more than once to obtain the thick layer of biopolymer as thick as $8 \mu \mathrm{m}-10 \mu \mathrm{m}$. The following method which may be able to increase the thickness of biopolymer layer was employed. The biopolymer was spincoated on the silicon wafer and then the buffer solution was evaporated in order to increase the viscosity of the spincoated biopolymeric layer. The clad layer with the more than $8 \mu \mathrm{m}$ thickness of the sample was obtained in order to meet the requirement of optical coupling and then the core layer in the $4 \mu \mathrm{m}$ thickness was spincoated on the clad layer to complete fabrication of the optical waveguide. Figure 8 shows surface quality of the coated biopolymer. A certain amount of conglomerated chitosan particles was observed. Figure 9 shows magnification of the photo shown in Figure 8.

Table 1. Cladding layer thickness.

\begin{tabular}{ccccc}
\hline $\begin{array}{c}\text { Spin-Coating } \\
\text { Velocity }(\mathrm{rpm})\end{array}$ & $\begin{array}{c}\text { Spin-Coating } \\
\text { Time }(\mathrm{s})\end{array}$ & $\begin{array}{c}\text { Thickness } \\
(\mu \mathrm{m})\end{array}$ & $\begin{array}{c}\text { Dry } \\
\text { Temperature }\left({ }^{\circ} \mathrm{C}\right)\end{array}$ & $\begin{array}{c}\text { Dry Time } \\
(\mathrm{hr})\end{array}$ \\
\hline 500 & 30 & 0.640 & 50 & 5 \\
500 & 30 & 4.8 & 50 & 5 \\
\hline
\end{tabular}

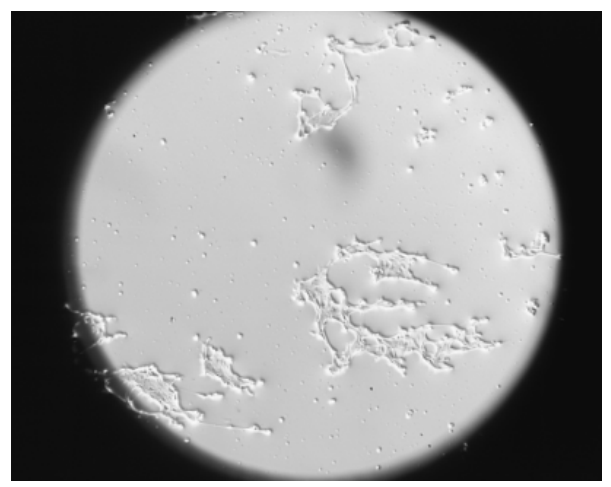

Figure 8. Photo of the surface of biopolymer.

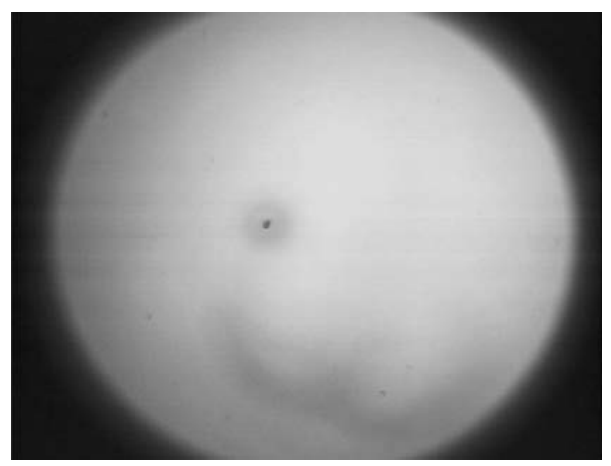

Figure 9. Photo of the sample is produced. 
The ellipsometer was used to measure the refractive indices of biopolymer layers. As a result, the measured refractive indices are $n=1.516$ for the clad layer and $n=$ 1.520 for the core layer, respectively, as shown in Figure 10. Change in the ratio of chitosan and gelatin enables us adjust the refractive index.

The clad thickness of the sample waveguide, which was coupled with the light, was more than $8.5 \mu \mathrm{m}$, and the core thickness of the sample was more than $4.5 \mu \mathrm{m}$. The transmitted light through the waveguide was measured by using the CCD camera, as shown in Figure 11.

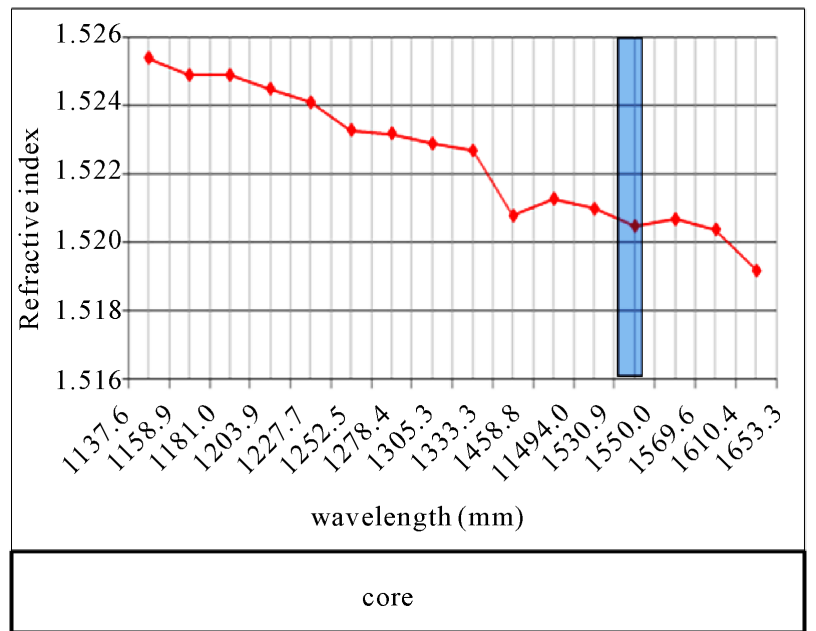

\section{Conclusions}

The biopolymric optical planar waveguide and Bragg grating were developed in this study. Gelatin and chitosan were blended with the proper ratio to develop the biopolymers with the different refractive index. The refractive indices of the spincoated biopolymer clad and core layers of the waveguide were obtained to be used in the planar waveguide. Then, the Bragg grating was successfully imprinted on the biopolymeric waveguide. The biopolymeric planar waveguide Bragg grating, which is biocompatible, implantable, and biodegradable, will have

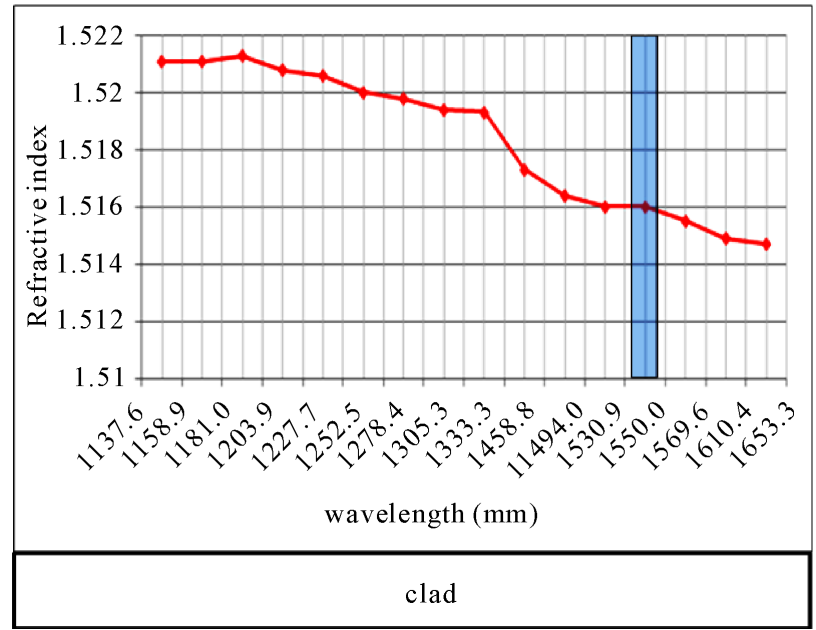

Figure 10. Meausred refractive index of biopolymer by using the ellipsometer.

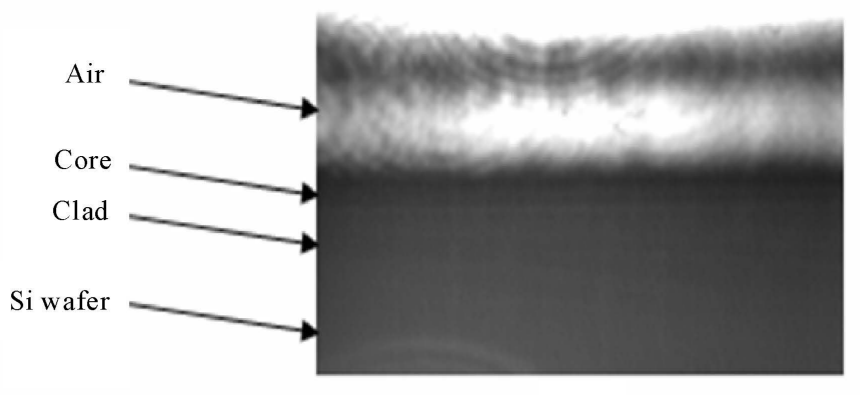

Air

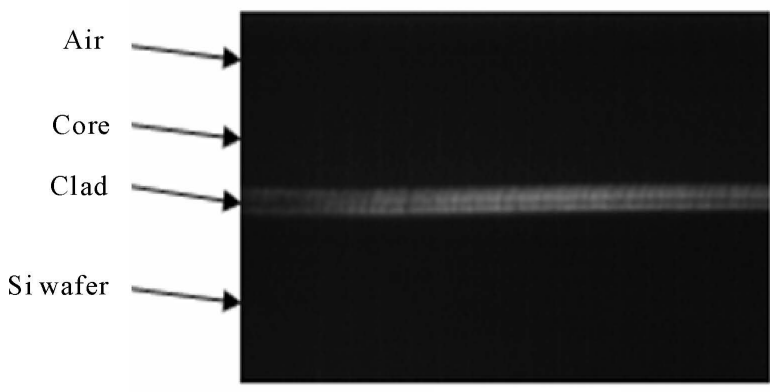

Clad

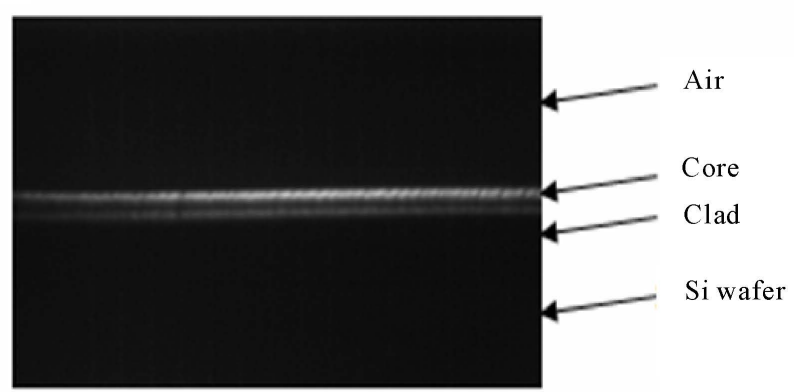

Core

Figure 11. CCD image for light coupling measurements. 

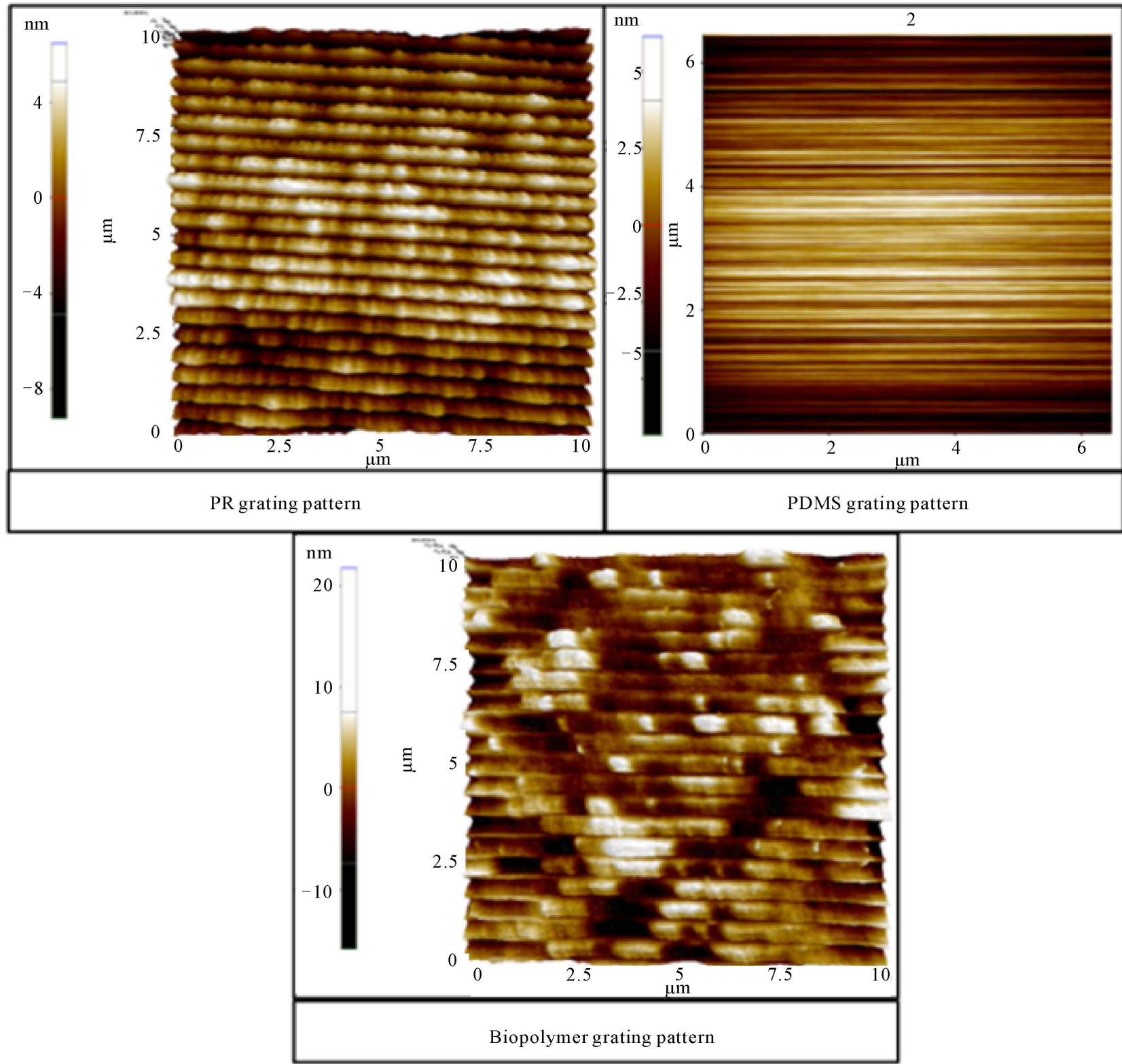

Figure 12. AFM images of the imprinted grating on the biopolymeric waveguide.

a great potential in application for biomedical diagnosis and monitoring as well as military and environmental

\section{Acknowledgements}

This work was supported by the National Research Foundation Korea Grant funded by the Korean Government (MEST) (NRF-2009-0076655).

\section{REFERENCES}

[1] K. C. Basavaraju, T. Damappa and S. K. Rai, "Preparation of Chitosan and Its Miscibility Studies with Gelatin Using Viscosity, Ultrasonic And Refractive Index," Carbohydrate Polymers, Vol. 66, No. 3, 2006, pp. 357-362. doi:10.1016/j.carbpol.2006.03.020

[2] W. Ding, Q. Lian, R. J. Samuels and M. B. Polk, "Synthesis and Characterization of a Novel Derivative of Chitosan,” Polymer, Vol. 44, No. 3, 2003, pp. 547-556. doi:10.1016/S0032-3861(02)00834-0

[3] J. C. Martinez-Anton and E. Bernabeu, "Spectrogoniometry and the WANTED Method for Thickness and Refractive Index Determination,” Thin Solid Film, Vol. 313, 1998, pp. 85-89. doi:10.1016/S0040-6090(97)00775-X

[4] H. Jiang, W. Su, S. Caracci, T. I. Bunning, T. Cooper, and W. W. Adams, "Optical Waveguiding and Morphology of Chitosan Thin Films," Journal of Applied Polymer Science, Vol. 61, No. 7, 1996, pp. 1163-1171. 
doi:10.1002/(SICI)1097-4628(19960815)61:7<1163::AID -APP12>3.0.CO;2-Z

[5] K.C. Basavaraju, T. Damappa and S. K. Rai,; "Preparation of Chitosan and Its Miscibility Studies with Gelatin Using Viscosity, Ultrasonic and Refractive Index,” Car- bohydrate Polymers, Vol. 66, No. 3, 2006, pp. 357-362. doi:10.1016/j.carbpol.2006.03.020

[6] A. Yariv, "Coupled-Mode Theory for Guided - Wave Optics,” IEEE Journal of Quantum Electronics, Vol. 9, No. 9, 1973, pp. 919-933. doi:10.1109/JQE.1973.1077767 